\title{
Physiotherapists Have Accurate Expectations of Their Patients' Future Health-Related Quality of Life after First Assessment in a Subacute Rehabilitation Setting
}

\author{
Steven M. McPhail, ${ }^{1,2}$ Emily Nalder, ${ }^{1,2,3}$ Anne-Marie Hill, ${ }^{4}$ and Terry P. Haines ${ }^{5,6}$ \\ ${ }^{1}$ Centre for Functioning and Health Research, Buranda Plaza Corner Ipswich Road and Cornwall Street, Buranda, QLD, Australia \\ ${ }^{2}$ School of Public Health and Institute of Health and Biomedical Innovation, Queensland University of Technology, Kelvin Grove, \\ QLD, Australia \\ ${ }^{3}$ Rotman Research Institute, Bathurst Street, Baycrest, Toronto, ON, Canada \\ ${ }^{4}$ School of Physiotherapy, the University of Notre Dame Australia, Mouat Street, Fremantle, WA, Australia \\ ${ }^{5}$ Southern Health, Allied Health Research Unit, Kingston Centre, Cnr Warrigal and Kingston Roads, Cheltenham, VIC, Australia \\ ${ }^{6}$ Physiotherapy Department, School of Primary Health Care, Monash University, Monash University Peninsular Campus, \\ VIC, Australia
}

Correspondence should be addressed to Steven M. McPhail; steven.mcphail@health.qld.gov.au

Received 30 April 2013; Accepted 19 September 2013

Academic Editor: Michael Rapp

Copyright (C) 2013 Steven M. McPhail et al. This is an open access article distributed under the Creative Commons Attribution License, which permits unrestricted use, distribution, and reproduction in any medium, provided the original work is properly cited.

Background. Expectations held by health professionals and their patients are likely to affect treatment choices in subacute inpatient rehabilitation settings for older adults. There is a scarcity of empirical evidence evaluating whether health professionals expectations of the quality of their patients' future health states are accurate. Methods. A prospective longitudinal cohort investigation was implemented to examine agreement (kappa coefficients, exact agreement, limits-of-agreement, and intraclass-correlation coefficients) between physiotherapists' $(n=23)$ prediction of patients' discharge health-related quality of life (reported on the EQ-5D-3L) and the actual health-related quality of life self-reported by patients $(n=272)$ at their discharge assessment (using the EQ-5D-3L). The mini-mental state examination was used as an indicator of patients' cognitive ability. Results. Overall, 232 (85\%) patients had all assessment data completed and were included in analysis. Kappa coefficients (exact agreement) ranged between $0.37-0.57(58 \%-83 \%)$ across EQ-5D-3L domains in the lower cognition group and $0.53-0.68(81 \%-85 \%)$ in the better cognition group. Conclusions. Physiotherapists in this subacute rehabilitation setting predicted their patients' discharge health-related quality of life with substantial accuracy. Physiotherapists are likely able to provide their patients with sound information regarding potential recovery and health-related quality of life on discharge. The prediction accuracy was higher among patients with better cognition than patients with poorer cognition.

\section{Background}

Many older adults do not return to their premorbid health states following hospital admissions requiring a period of subacute inpatient rehabilitation [1-4]. Patient-centred models of care are becoming increasingly common among subacute rehabilitation facilities [5-7]. Patient-centred models of care may assist older adults recovering from illness or injury to work with their treating team to focus therapy and other treatments on deficits that are most important to the patient to optimise their health-related quality of life at discharge or thereafter $[5,6]$. It may therefore be beneficial for patients to have an accurate understanding of their likely recovery in order to make informed decisions about joint therapy goals and when planning for living in the community after discharge $[5,6]$.

There is currently a scarcity of research investigating whether health-professionals' and patients' expectations of 
future health-states are accurate among patient groups receiving health interventions in settings that utilise patient centred models of care [8]. A recent investigation among older adults in a subacute hospital setting indicated that patients' expectations of their discharge health-related quality of life were moderately accurate [8]. In this context it is likely that older patients will derive information regarding their current health state and potential patterns of recovery from their treating healthcare team [8]. Unfortunately, no previous studies have investigated whether health professionals are able to formulate accurate predictions of older patients' discharge health-related quality of life in subacute hospital settings.

Physiotherapists are one group of health professionals of particular interest in subacute hospital settings where older adults receive rehabilitative therapies to address functional concerns in order to maximize health-related quality of life on discharge from hospital [2, 9-12]. It is likely that older adults participating in rehabilitative therapies to improve their physical function will derive guidance from their physiotherapists when planning functional goals in this setting. However, there is currently no prior research investigating whether physiotherapists are able to formulate accurate predictions of patients' discharge health states early in a subacute rehabilitation admission. Accurate expectations of their older patients' potential (or lack of potential) to improve their functioning and health-related quality of life during a subacute rehabilitation admission are likely to be beneficial to patients, therapists, and other members of the multidisciplinary team involved in planning clinical interventions as well as planning for discharge $[5-8,11,13,14]$.

This study investigated whether physiotherapists' expectations of their patients' discharge health-related quality of life (formulated while conducting their admission assessment) were in agreement with their patients' actual self-reported health-related quality of life on discharge from a subacute rehabilitation unit. Due to the potential influence of patient cognition on self-reported health-related quality of life at discharge, the levels of agreement between physiotherapist predicted health-related quality of life and patients' selfreported discharge health-related quality of life were examined for an entire cohort, as well as two cognition subgroups based on mini-mental state examination scores [15-17].

\section{Methods}

2.1. Design. A prospective longitudinal cohort design was implemented to examine agreement between physiotherapists' expectations of their patients discharge health-related quality of life versus the patients' actual discharge healthrelated quality of life as self-reported on the EQ-5D-3L [18].

2.2. Participants and Setting. A total of 272 patients and their treating physiotherapists $(n=23)$ from a subacute geriatric assessment and rehabilitation unit at tertiary hospital participated in this investigation. Participants were consecutive admissions to this unit following a period of acute management on a specialised hospital ward appropriate for their condition (e.g., an acute orthopaedic ward). The participating unit admits subacute older adults with a range of primary diagnoses for rehabilitative interventions to improve their functioning and prepare them for discharge from hospital. Patients admitted to the participating rehabilitation unit received medical and allied health input designed to maximize functioning, independence, and health-related quality of life on discharge. The typical length of stay in this rehabilitation unit at the time of the study was approximately six weeks. However, length of stay was determined on an individual basis and may have been less or more than six weeks dependent on a range of clinical and external factors.

2.3. Outcome Measure. The EQ-5D-3L [18] instrument used to evaluate health-related quality of life has favourable reliability and validity for use with older people [19-24]. This instrument has six questions. The first five items require individuals to rate their health-related quality of life in the domains of mobility, personal care, usual activities, pain and discomfort, and anxiety and depression on a threelevel multiple choice scale ((1) no problems, (2) some problems/moderate, and (3) unable/extreme problems). The brief multiple choice scoring system was deemed suitable due to its ease of administration and no requirement for high levels of health literacy in order to understand and respond to each question. The Dolan tariff system [25] was applied to responses to the first five EQ-5D-3L items producing a multiattribute utility score (utility) where death and perfect health are represented by 0 and 1 , respectively. Health states considered worse than death are assigned negative values [25]. The sixth and final EQ-5D-3L question is a 100 point Visual Analogue Scale (EQ-VAS) where 0 and 100 are represented by worst and best imaginable health state, respectively $[18,26]$.

The physiotherapists working in the participating subacute rehabilitation unit routinely complete clinical assessments within 72 hours of admission for their patients. It was at the completion of this routine admission assessment that physiotherapists in this unit predicted patients' discharge health-related quality of life. They did this by filling out an EQ-5D-3L selecting the responses they anticipated their patient would self-report during the discharge assessment.

The Mini-mental state examination (MMSE) was used as a general indicator of patient cognitive ability [16]. The MMSE incorporates a brief assessment of orientation, memory, attention, and arithmetic and is routinely completed for all patients in the participating clinical unit [16, 27]. The MMSE was used to separate patients for subgroup analyses of a poorer cognition group (MMSE of 23 or less) and a better cognition group (MMSE greater than 23) [16, 27].

2.4. Procedure. On admission and discharge from the unit, patients completed a standard battery of clinical assessments. This assessment included physical performance tests (such as Balance Outcome Measure for Elder Rehabilitation [9]) as well as self-ratings on the EQ-5D-3L to obtain the patient's current perceived health-related quality of life (at admission to the unit). 
TABLE 1: Participant demographic and clinical information.

\begin{tabular}{lcc}
\hline & Low cognition group $(n=81)$ & High cognition group $(n=151)$ \\
\hline Mean (SD) Age & $79.0(11.8)$ & $71.7(14.9)$ \\
Gender-Female (\%) & $51(63 \%)$ & $88(58 \%)$ \\
Clinical diagnosis category at admission (\%) & & $45(30 \%)$ \\
$\quad$ Orthopedic & $30(37 \%)$ & $41(27 \%)$ \\
Stroke & $12(15 \%)$ & $24(16 \%)$ \\
Other Neurological & $11(14 \%)$ & $14(9 \%)$ \\
Geriatric deconditioning & $12(15 \%)$ & $27(18 \%)$ \\
Other disabling condition requiring rehabilitation & $16(20 \%)$ & $42(26-70)$ \\
Median (IQR) days length of stay & $45(25-65)$ & $0.425(0.352)$ \\
EQ-5D-3L at admission & & $57(19)$ \\
Mean (SD) Utility Score & $0.444(0.402)$ & $63(19)$ \\
Mean (SD) VAS & & \\
\hline
\end{tabular}

Immediately following the completion of the admission clinical assessment battery (but before patients completed their admission EQ-5D-3L self-report), physiotherapy staff completed their discharge prediction rating on the EQ-5D$3 \mathrm{~L}$ instrument. Physiotherapist participants received a 1-hour training session regarding their role in this study, including how to follow the assessment protocols at the admission and discharge assessments. The training included completing a practice admission and discharge assessment under the supervision of a member of the research team. Additionally, instructions for providing the predicted discharge EQ-5D$3 \mathrm{~L}$ report were included in each patient's chart. As part of the training session, therapists were instructed to rate the EQ-5D-3L according to how they believed the patient would answer EQ-5D-3L items on discharge from rehabilitation, regardless of whether clinicians agreed with the patient's perspective.

The MMSE was completed by hospital occupational therapists or medical staff for each patient admitted to the unit. MMSE results along with other patient demographic variables were collated from the medical history, and patients were grouped into a better cognition group (MMSE >23/30) and a poorer cognition group (MMSE $\leq 23 / 30$ ) for use in data analysis.

This research investigation was approved by the local human research ethical review board and complied with the declaration of Helsinki. Patients completed the EQ-5D$3 \mathrm{~L}$ at discharge as a component of their routine clinical assessments, and gatekeeper consent was provided for physiotherapists' participation in this research.

2.5. Analysis. Demographic and clinical characteristics of the sample were tabulated (Table 1). Conventional tests of hypothesis were used to compare demographic characteristics between the lower and higher cognition groups. This included an unpaired $t$-test (age) and a Mann-Whitney $U$ test (length of stay in the subacute rehabilitation unit). For the individual EQ-5D-3L domain scores, levels of agreement between physiotherapist predicted discharge rating and actual self-reported discharge EQ-5D-3L responses were calculated using weighted Cohen's Kappa with disagreements of only one level assigned a 0.5 weighting [28] (Table 2); bias corrected $95 \%$ confidence intervals for Kappa coefficients were calculated using bootstrap resampling (2000 replications of original sample size, stratifying for cognition grouping where appropriate) $[29,30]$. The number (and percentage) of exact matches for each of the domains was also tabulated (Table 2).

For the summary, EQ-5D-3L scores (utility and EQ-VAS), limits of agreement (LOA) [31], and intraclass-correlation coefficients (Table 3) were calculated separately for patients in each cognition grouping as well as for the total sample. To investigate systematic differences between therapist predicted and patients' discharge health-related quality of life scores (for utility and EQ-VAS) paired $t$-tests were employed separately for each cognition group as well as for the whole patient cohort (Table 3).

\section{Results}

Overall, $232(85 \%)$ patients had complete data and were included in analyses; 81 (35\%) were in the lower cognition group and 151 (65\%) were in the higher cognition group. The demographic and clinical characteristics of the sample are presented in Table 1. The poorer cognition group was older by a mean (SD) 7.3 (1.9) years than the better cognition group $(P<0.001)$. The median (IQR) length of stay for the entire sample was 42 (25-66), with no difference between cognition groups $(P=0.60)$. The median (interquartile range) years of experience of physiotherapists $(n=23)$ providing a prediction rating was 6 (2-11).

The levels of agreement (Kappa statistics and exact matches) between physiotherapist predicted discharge healthrelated quality of life (EQ-5D-3L domain scores) and patients' self-reported health-related quality of life (EQ-5D-3L domain scores) are reported in Table 2. Kappa coefficients ranged from 0.37 to 0.57 across domains in the lower cognition group and from 0.53 to 0.68 in the better cognition group. Exact agreement ranged from $58 \%$ to $83 \%$ across domains in the lower cognition group and from $81 \%$ to $85 \%$ in the 


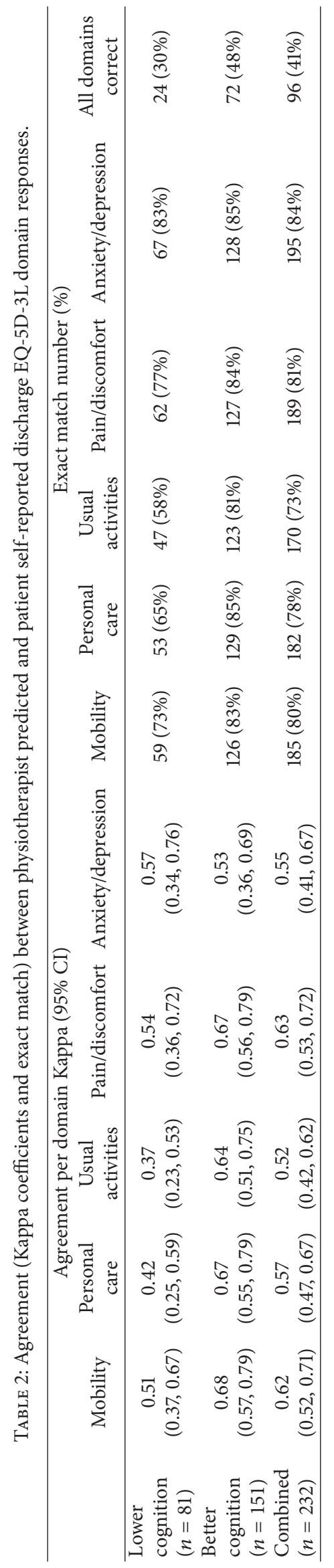




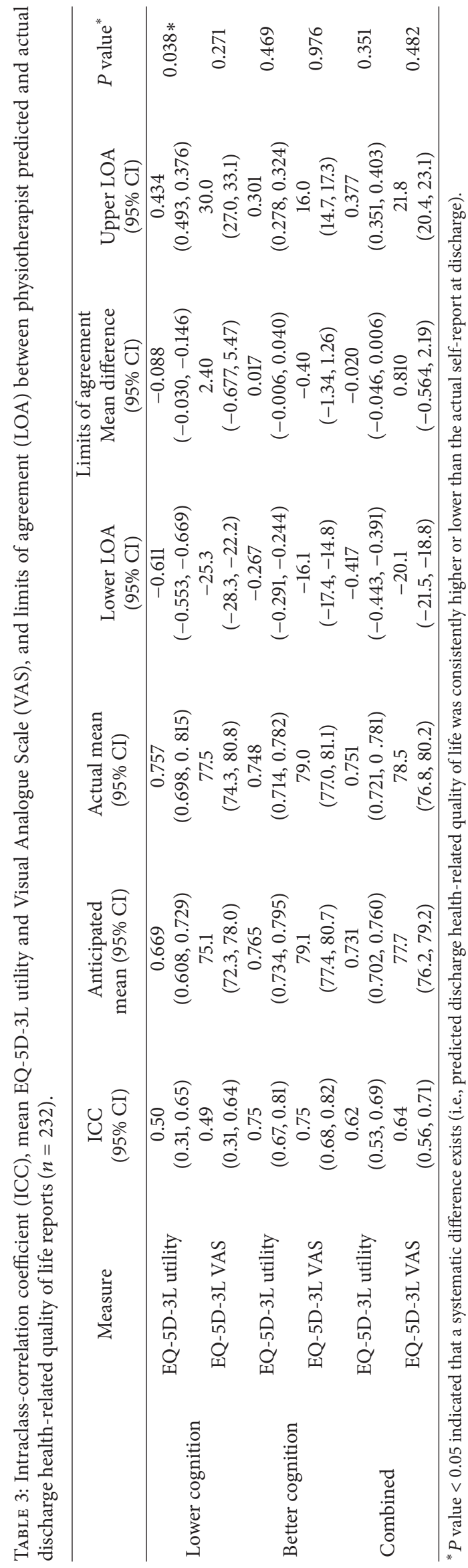


better cognition group. Overall, $41 \%$ of patients had all five discharge EQ-5D-3L domain responses correctly predicted by their treating physiotherapist at their baseline assessments.

Agreement (intraclass-correlation coefficients and limits of agreement) between physiotherapist predicted discharge health-related quality of life and patients' self-reported health related quality of life at discharge is displayed in Table 3 for the EQ-5D-3L utility and EQ-VAS. The better cognition group and entire sample had narrower limits of agreement and higher intraclass-correlation coefficients than the lower cognition group. No mean differences between predicted and actual discharge EQ-VAS scores were observed for either cognition group or for the entire patient sample as a whole. The mean predicted utility score was lower than the actual discharge utility score for the lower cognition group $(-0.09, P=0.038)$. No differences were observed in mean utility ratings (predicted versus actual) for the overall sample or for the better cognition group.

\section{Discussion}

This investigation revealed substantial agreement between physiotherapists' predictions of their patients' health-related quality of life at discharge and the patients' actual selfreported health-related quality of life at discharge. Interestingly, patient cognitive ability seemed to be a mediating factor in the accuracy of the prediction. The agreement between physiotherapist predicted and self-reported quality of life was not only lower for patients in the poorer cognition group, but physiotherapists tended to underestimate the level of health-related utility (EQ-5D-3L utility) that patients' in the lower cognition group would report at discharge. The size of this underestimation was of a sufficient magnitude to be considered clinically meaningful [32-34]. On the other hand, physiotherapists were able to predict how patients in the better cognition group would report their health-related quality of life at discharge, without any mean overestimation or underestimation and with a substantial degree of accuracy (Table 3). Physiotherapists also had exact matches for all five domain responses for a higher proportion of patients in the better cognition group than the better poorer cognition group (Table 2).

The purpose of this investigation was not to probe the cause of any underestimation for patients with poorer cognition. However, the authors speculate that there are at least two potential reasons why physiotherapists may have underestimated the summary utility score generated by the EQ-5D-3L instrument for patients with poorer cognitive abilities. First, the physiotherapists would have had some knowledge of patients' cognitive ability from reviewing their medical record and receiving a clinical handover of pertinent information from their colleagues prior to conducting their assessment in this subacute hospital setting. It is plausible that physiotherapists perceived a level of impact from the cognitive impairment on the patients' potential for recovery that was not accurate, and patients subsequently experienced more recovery and better health-related quality of life (EQ5D-3L utility score) at discharge than the physiotherapists had anticipated. Second, it is also plausible that patients from the poorer cognition group may have lacked some level of insight into their level of functioning when responding to the EQ-5D-3L questions at their discharge assessment. For example, a patient with poor cognition who had some problems with their ability to wash and dress themselves may have reported having no problems with personal care. Potential lack of insight of this nature may have led to difficulty predicting how patients might respond at their discharge assessment rather than difficulty predicting the level of functional recovery a patient may experience relevant to their health-related quality of life [17].

There was no clear pattern in the level of prediction accuracy across the five EQ-5D-3L domains for patients in the better cognition group, with the level of exact agreement consistently between $81 \%$ and $85 \%$ for all five domains. Similarly, there was no clear pattern in the level of prediction accuracy within the poorer cognition group across the five health-related quality of life domains, despite the agreement statistics being lower than those observed for the better cognition group. This was an interesting finding, given that some prior research has indicated that proxies may be better able to predict how patients will respond to health survey questions for more observable domains like mobility than for less easily observable domains like depression [35-37]. One could argue that physiotherapists, who have particular expertise in physical recovery, may have been more likely to predict health-related quality of life domains related to physical functioning than depression or anxiety. However, such an assertion would not have been supported by the empirical data from this investigation.

There are several important implications for patient centred models of care in subacute rehabilitation settings from this research. Patients that experience debilitating injuries or illnesses are likely to derive information about their potential for recovery from their treating health professionals when planning goals and making joint treatment decisions intended to maximize their health-related quality of life on discharge and thereafter $[5,6]$. Physiotherapists are likely to be a reliable source of information regarding patients' potential for recovery during the subacute rehabilitation period from as early as immediately following their initial assessment on admission to subacute rehabilitation units. However, further research is warranted to investigate whether clinicians tend to underestimate the potential for recovery among patients with poorer cognition or the extent to which patients with poor cognition may under-report potential deficits in their health-related quality of life when completing questionnaires.

There may be other explanatory factors beyond the scope of this investigation that influenced patient self-reports among some patients and contributed to some discrepancies between predicted and actual health-related quality of life reports in both cognition groups. Some previous reports have demonstrated paradoxical improvements in self-reported health-related quality of life as people have aged despite declining physical functioning and increased depression [3840]. Changes in the way people reconceptualize, reprioritize, and recalibrate their health state (ratings) as an effect of natural adaptation over time have been termed "response 
shift" and may have influenced the older adults in this population receiving rehabilitation [7, 24, 41]. Individual factors, such as these, that may cause a measurement related bias potentially influencing patient reports of health-related quality of life (and difficulty anticipating future health state reports) are worthy of consideration as a future research direction with relevance to patient centred models of care.

There are several strengths and limitations for this research. First, all participating physiotherapists and patients were from a single geographical location. Physiotherapists from other geographical locations may not have responded in the same way. On the other hand, the inclusion of 23 different physiotherapists and 224 patients provides some ability to generalize findings to other similar populations. Second, only the EQ-5D-3L instrument was used. While this instrument is among the most widely used generic health-related quality of life measures, is suitable for use among older adults [19, 23], and is suited to this kind of predictive study due to the clearly delineated response options, use of another method of healthrelated quality of life reporting may not have yielded the same levels of predictive accuracy. Another important caveat is that this investigation only included patients in the subacute inpatient rehabilitation phase of their recovery. It is possible that physiotherapists answered some of the domain responses knowing the minimum level of functioning that would be required for patients to be able to be safely discharged and provided predictive responses congruent with minimum requirements for safe discharge to community living rather than basing their specific responses on knowledge of the pathology, the patient, and their prognosis. Nonetheless, this investigation was successful in addressing the research aims.

It may be useful for future research in this field to examine the prediction of health state outcomes across transition periods. This may include transitions from inpatient rehabilitation to returning to live in the community, particularly among older patients at risk of requiring institutional care. Intervention decisions in the subacute setting may influence the likelihood of subsequent hospital or nursing care admissions. The ability for clinical teams to be able to inform their patients about the likelihood of functional abilities and health-related quality of life in the post-discharge period may assist patients (in conjunction with their treating teams) to make sound decisions regarding postdischarge care arrangements. Additionally, specific physiotherapist-related factors that may have influenced their ability to predict patients' discharge health-related quality of life were beyond the scope of this investigation. However, future investigations of health-professional attributes that may influence their ability to anticipate their patients' future health states may also be worthy of consideration to advance this field of research.

\section{Conclusion}

Physiotherapists in this subacute rehabilitation setting predicted their patients' discharge health-related quality of life with substantial accuracy. They are likely to be able to provide their patients with sound information regarding the potential recovery and health-related quality of life on discharge. The prediction accuracy was higher among patients with better cognition than patients with poorer cognition. Physiotherapists tended to underestimate the self-reported discharge health-related quality of life of patients in the poorer cognition group by a small, but potentially clinically meaningful, margin.

\section{Conflict of Interests}

The authors declare that they have no conflict of interests.

\section{Authors' Contribution}

Steven M. McPhail contributed to research idea conception, data collection, data analysis, and paper preparation, as well as paper review, appraisal, and editing. Emily Nalder contributed to paper drafting, editing, and appraisal. Terry P. Haines contributed to research idea conception and paper review, appraisal, and editing. All authors read and approved the final paper.

\section{Acknowledgments}

The authors would like to acknowledge the valuable contribution from the staff and patients involved in this research. Steven M. McPhail, Anne-Marie Hill, and Terry P. Haines are supported by the National Health and Medical Research Council (of Australia) fellowships.

\section{References}

[1] S. McPhail, E. Beller, and T. Haines, "Physical function and health-related quality of life of older adults undergoing hospital rehabilitation: how strong is the association?" Journal of the American Geriatrics Society, vol. 58, no. 12, pp. 2435-2437, 2010.

[2] K. Burgess, S. Kuys, S. McPhail, and P. Varghese, "Outcomes following inpatient rehabilitation: a seven year perspective," Australasian Journal on Ageing, vol. 31, p. 9, 2012.

[3] S. McPhail, K. Burgess, S. Kuys, and P. Varghese, "Patient stay in a geriatric rehabilitation unit: 2011 versus 2005," Australasian Journal on Ageing, vol. 31, p. 33, 2012.

[4] L. C. Giles, G. Hawthorne, and M. Crotty, "Health-related quality of life among hospitalized older people awaiting residential aged care," Health and Quality of Life Outcomes, vol. 7, article 71, 2009.

[5] M. van der Eijk, M. J. Faber, S. Al Shamma, M. Munneke, and B. R. Bloem, "Moving towards patient-centered healthcare for patients with Parkinson's disease," Parkinsonism and Related Disorders, vol. 17, no. 5, pp. 360-364, 2011.

[6] L. Sandman and C. Munthe, "Shared decision making, paternalism and patient choice," Health Care Analysis, vol. 18, no. 1, pp. 60-84, 2010.

[7] S. McPhail, T. Comans, and T. Haines, "Evidence of disagreement between patient-perceived change and conventional longitudinal evaluation of change in health-related quality of life among older adults," Clinical Rehabilitation, vol. 24, no. 11, pp. 1036-1044, 2010.

[8] S. McPhail and T. Haines, "Patients undergoing subacute rehabilitation have accurate expectations of their health-related 
quality of life at discharge," Health and Quality of Life Outcomes, vol. 10, no. 1, p. 94, 2012.

[9] T. Haines, S. S. Kuys, G. Morrison, J. Clarke, P. Bew, and S. McPhail, "Development and validation of the balance outcome measure for elder rehabilitation," Archives of Physical Medicine and Rehabilitation, vol. 88, no. 12, pp. 1614-1621, 2007.

[10] A. M. Hill, K. Hill, S. Brauer et al., "Evaluation of the effect of patient education on rates of falls in older hospital patients: description of a randomised controlled trial," BMC Geriatrics, vol. 9, no. 1, article 14, 2009.

[11] A. M. Hill, T. Hoffmann, S. McPhail et al., "Factors associated with older patients' engagement in exercise after hospital discharge," Archives of Physical Medicine and Rehabilitation, vol. 92, no. 9, pp. 1395-1403, 2011.

[12] H. Batten, S. Kuys, S. McPhail, J. Nitz, and P. Varghese, "Outcomes following inpatient rehabilitation of lower limb amputees," Australasian Journal on Ageing, vol. 31, p. 68, 2012.

[13] A. M. Hill, T. Hoffmann, C. Beer et al., "Falls after discharge from hospital: is there a gap between older peoples' knowledge about falls prevention strategies and the research evidence?" Gerontologist, vol. 51, no. 5, pp. 653-662, 2011.

[14] A. M. Hill, A. Hoffmann, S. McPhail et al., "Evaluation of the sustained effect of inpatient falls prevention education and predictors of falls after hospital discharge-follow-up to a randomized controlled trial," Journals of Gerontology A, vol. 66, no. 9, pp. 1001-1012, 2011.

[15] B. Agrell and O. Dehlin, "Mini mental state examination in geriatric stroke patients. Validity, differences between subgroups of patients, and relationships to somatic and mental variables," Aging, vol. 12, no. 6, pp. 439-444, 2000.

[16] M. F. Folstein, S. E. Folstein, and P. R. McHugh, “Mini mental state. A practical method for grading the cognitive state of patients for the clinician," Journal of Psychiatric Research, vol. 12, no. 3, pp. 189-198, 1975.

[17] S. McPhail, E. Beller, and T. Haines, "Two perspectives of proxy reporting of health-related quality of life using the euroqol-5D, an investigation of agreement," Medical Care, vol. 46, no. 11, pp. 1140-1148, 2008.

[18] R. Rabin and F. de Charro, "EQ-5D: a measure of health status from the EuroQol group," Annals of Medicine, vol. 33, no. 5, pp. 337-343, 2001.

[19] S. J. Coons, S. Rao, D. L. Keininger, and R. D. Hays, "A comparative review of generic quality-of-life instruments," Pharmacoeconomics, vol. 17, no. 1, pp. 13-35, 2000.

[20] M. Fransen and J. Edmonds, "Reliability and validity of the EuroQol in patients with osteoarthritis of the knee," Rheumatology, vol. 38, no. 9, pp. 807-813, 1999.

[21] P. F. M. Krabbe, L. Peerenboom, B. S. Langenhoff, and T. J. M. Ruers, "Responsiveness of the generic EQ-5D summary measure compared to the disease-specific EORTC QLQ C-30," Quality of Life Research, vol. 13, no. 7, pp. 1247-1253, 2004.

[22] B. Schweikert, H. Hahmann, and R. Leidl, "Validation of the EuroQol questionnaire in cardiac rehabilitation," Heart, vol. 92, no. 1, pp. 62-67, 2006.

[23] S. McPhail, P. Lane, T. Russell et al., "Telephone reliability of the Frenchay activity index and EQ-5D amongst older adults," Health and Quality of Life Outcomes, vol. 7, article 48, 2009.

[24] S. McPhail and T. Haines, "Response shift, recall bias and their effect on measuring change in health-related quality of life amongst older hospital patients," Health and Quality of Life Outcomes, vol. 8, no. 1, article 65, 2010.
[25] P. Dolan and J. Roberts, "Modelling valuations for Eq-5d health states: an alternative model using differences in valuations," Medical Care, vol. 40, no. 5, pp. 442-446, 2002.

[26] S. McPhail, E. Beller, and T. Haines, "Reference bias: presentation of extreme health states prior to eq-vas improves healthrelated quality of life scores. A randomised cross-over trial," Health and Quality of Life Outcomes, vol. 8, article 146, 2010.

[27] T. N. Tombaugh and N. J. McIntyre, "The mini-mental state examination: a comprehensive review," Journal of the American Geriatrics Society, vol. 40, no. 9, pp. 922-935, 1992.

[28] S. O.'Leary, M. Lund, T. J. Ytre-Hauge et al., "Pitfalls in the use of kappa when interpreting agreement between multiple raters in reliability studies," Physiotherapy, 2013.

[29] B. Efron and R. Tibshirani, An Introduction to the Bootstrap, Chapman and Hall, New York, NY, USA, 1993.

[30] J. Lee and K. P. Fung, "Confidence interval of the kappa coefficient by bootstrap resampling," Psychiatry Research, vol. 49, no. 1, pp. 97-98, 1993.

[31] J. M. Bland and D. G. Altman, "Statistical methods for assessing agreement between two methods of clinical measurement," The Lancet, vol. 1, no. 8476, pp. 307-310, 1986.

[32] G. Coteur, B. Feagan, D. L. Keininger, and M. Kosinski, "Evaluation of the meaningfulness of health-related quality of life improvements as assessed by the SF-36 and the EQ-5D VAS in patients with active Crohn's disease," Alimentary Pharmacology and Therapeutics, vol. 29, no. 9, pp. 1032-1041, 2009.

[33] N. Luo, L. H. Chew, K. Y. Fong et al., "Do English and Chinese EQ-5D versions demonstrate measurement equivalence? An exploratory study," Health and Quality of Life Outcomes, vol. 1, no. 1, article 7, 2003.

[34] A. S. Pickard, M. P. Neary, and D. Cella, "Estimation of minimally important differences in EQ-5D utility and VAS scores in cancer," Health Qual Life Outcomes, vol. 5, article 70, 2007.

[35] E. M. Andresen, V. J. Vahle, and D. Lollar, "Proxy reliability: health-related quality of life (HRQoL) measures for people with disability," Quality of Life Research, vol. 10, no. 7, pp. 609-619, 2001.

[36] K. C. A. Sneeuw, M. A. G. Sprangers, and N. K. Aaronson, "The role of health care providers and significant others in evaluating the quality of life of patients with chronic disease," Journal of Clinical Epidemiology, vol. 55, no. 11, pp. 1130-1143, 2002.

[37] P. J. Dorman, F. Waddell, J. Slattery, M. Dennis, and P. Sandercock, "Are proxy assessments of health status after stroke with the EuroQol questionnaire feasible, accurate, and unbiased?" Stroke, vol. 28, no. 10, pp. 1883-1887, 1997.

[38] G. L. Albrecht and P. J. Devlieger, "The disability paradox: high quality of life against all odds," Social Science and Medicine, vol. 48, no. 8, pp. 977-988, 1999.

[39] G. Netuveli and D. Blane, "Quality of life in older ages," British Medical Bulletin, vol. 85, no. 1, pp. 113-126, 2008.

[40] P. J. Allison, D. Locker, and J. S. Feine, "Quality of life: a dynamic construct," Social Science and Medicine, vol. 45, no. 2, pp. 221230, 1997.

[41] M. A. G. Sprangers and C. E. Schwartz, "Integrating response shift into health-related quality of life research: a theoretical model," Social Science and Medicine, vol. 48, no. 11, pp. 15071515, 1999. 


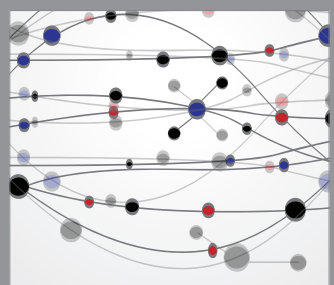

The Scientific World Journal
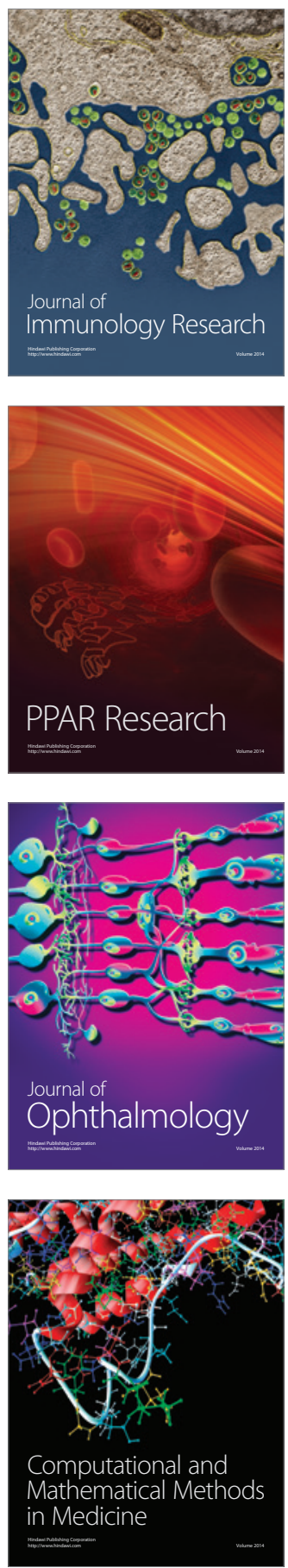

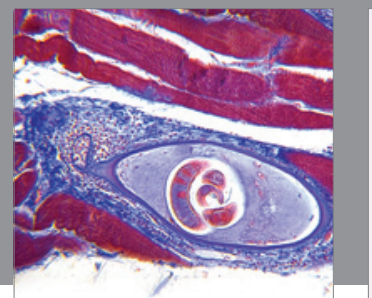

Gastroenterology

Research and Practice
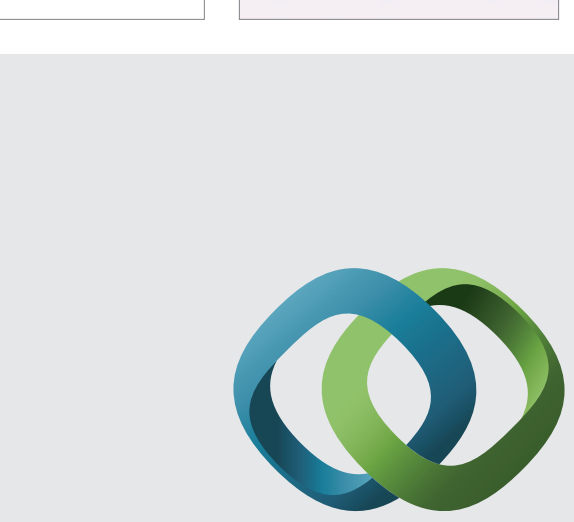

\section{Hindawi}

Submit your manuscripts at

http://www.hindawi.com
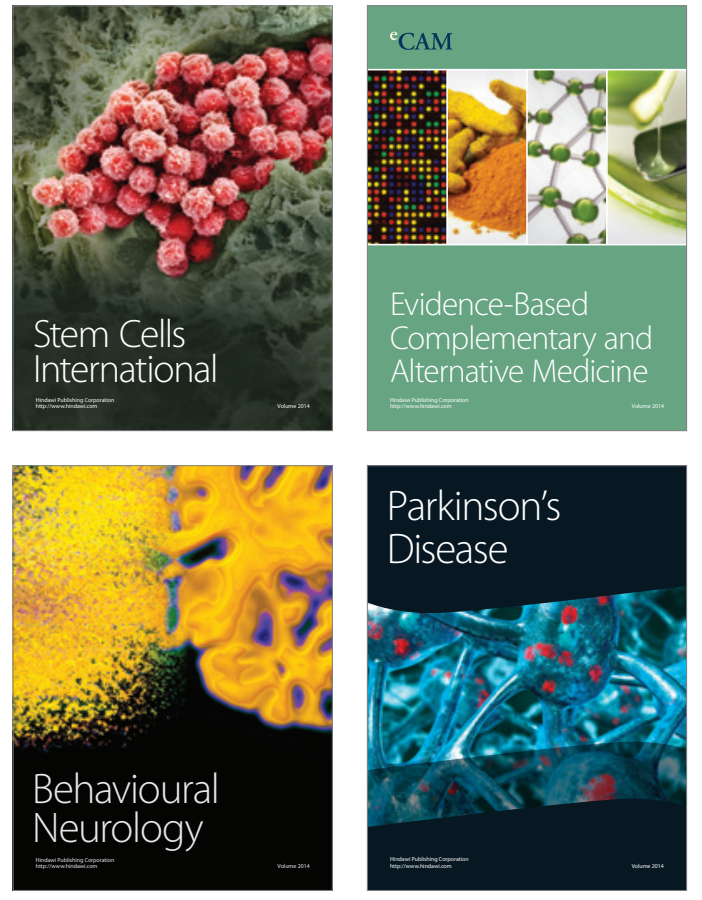
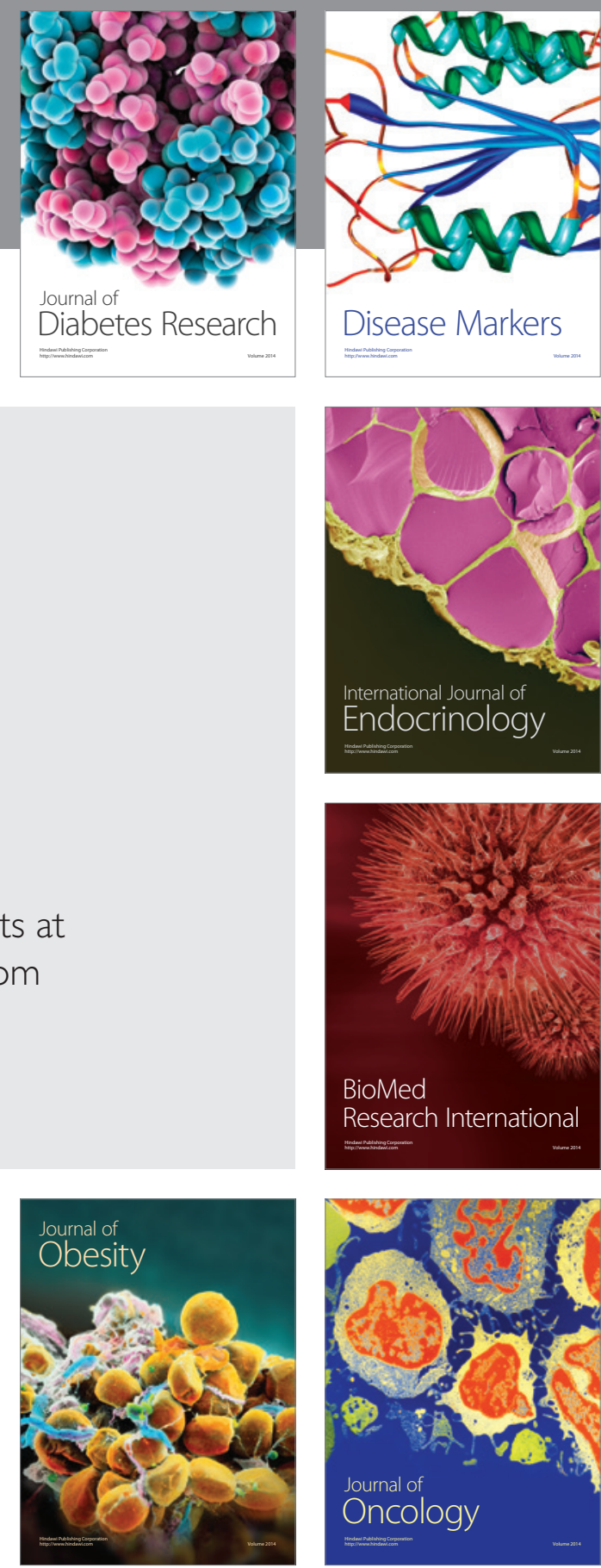

Disease Markers
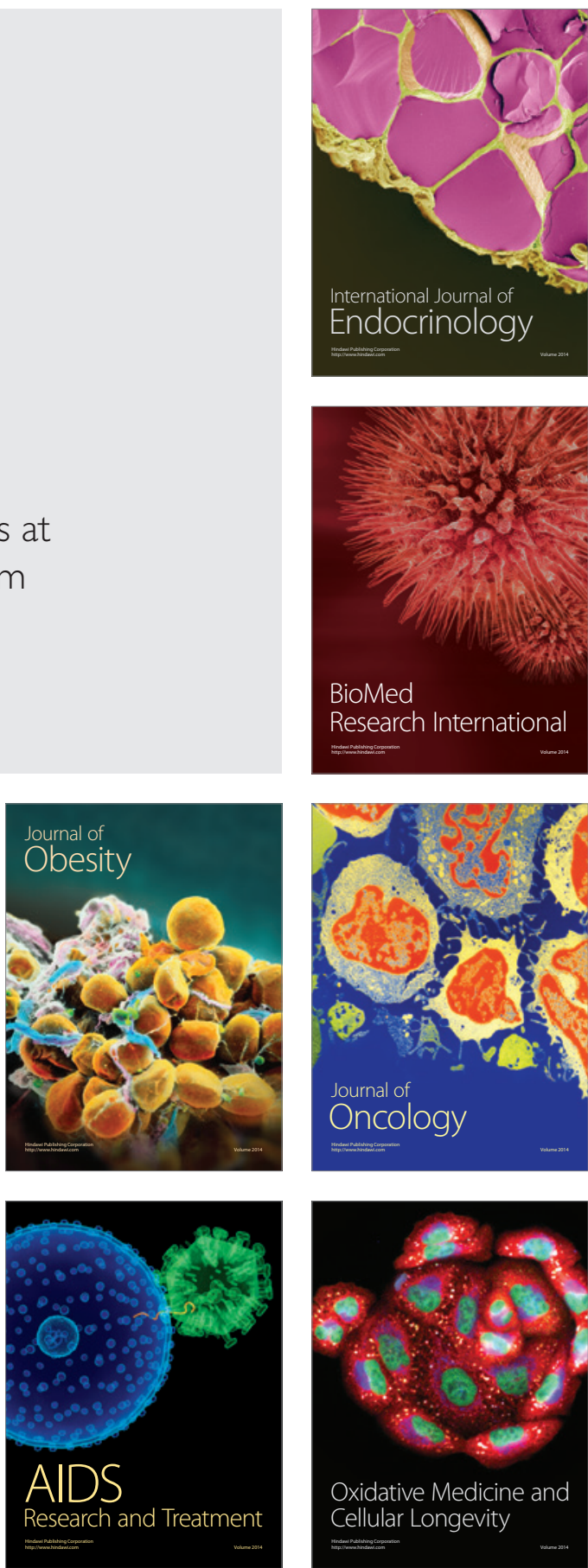